\title{
Mines Safety and Accident Communication System for underground mines
}

\author{
By \\ BBesa $^{1 *}$, Mulenga²and C Mazimba ${ }^{3}$ \\ 1, 2\&3 University of Zambia, School of Mines, Department of Mining Engineering, P.O. Box 32379, Lusaka, \\ Zambia
}

Email: bundabesa@yahoo.com or bbesa@unza.zm

\begin{abstract}
All mines have safety problems and underground mines tend to be thought of as more dangerous than surface mines. Safety communication systems typically found in Zambian underground mines include hardened telephone systems coupled with the Locked-Bell System, mine radios, leaky feeder systems and on a limited basis, digital systems. These systems depend upon a hard-wired support and if communications cable(s) become damaged or severed at any point, all communications in and out of an underground mine are disrupted. Additionally, fire, explosion or water inundation is likely to compromise communications cables and as such they cannot be relied upon for emergency communication purposes. These challenges lead to safety communication underground difficult and sometimes results in fatality when an accident happens. In this study, therefore, communications software is developed for underground mines to improve safety communication. An accident reporting and logging database based system is designed which shall incorporate the digital version of Form 55 used at Ministry of Mines and Minerals Development in Zambia. The communication software is developed using Real Basic programming language which is an object-oriented invent driven Language and Structured Query Language (SQL), a database management design and maintenance language. The communications software was developed and tested on a virtual and actual server-client networks i.e. it provided a two-way texting and chatting system, which is useful in relaying of information between the surface and teams working underground. The clients to be deployed underground will have a unique ID which can be referenced to give details on the locations. This can further ease the search for the miners in case of total loss of communication and accidents. The Mines Safety and Accident Communication System (MISACOMS) Reporting and Logging system was developed and tested on various operating systems. The digital format of Form 55 provided an ease way of logging, reporting and cross referencing accident data as it is stored in a database.
\end{abstract}

Keywords: Accident, Safety, Communication, Software, Underground

\section{INTRODUCTION}

All mines have safety problems, but underground mines tend to be thought of as especially dangerous. The danger stems from the fact that underground mine are constructed within natural rock which is a bad engineering material. As a result, underground mines are statistically more hazardous than surface mines. The major causes of accidents in most underground mines are falls of ground, either from the roof or sides of an excavation or material handling equipment, moving machinery and explosives. In a case of coal underground mines, methane explosion is a major cause of accidents. Most of the mining accidents are related to human error. In Zambian underground mines, hardened telephone systems coupled with the Locked-Bell System are used for communication between personnel underground and personnel on 
surface. Locked bell system is only used for communication at the shaft bank between underground personnel, cage tender and the winder driver. Hardened telephone systems are installed in each and every section, workshops and at the shaft stations. In most instances, as development and mining activities progress, working areas tend to move away from the section stations. This tends to make the phones not available at the production sites and other work areas. In an event where an accident happens, the workers at the scene of the accident need to get to the phone stations nearest to the section offices for them to call for help to aid the injured workmate(s). In the case were the accident has not been reported, the crashing point staff will be able to indicate which personnel has not made it to surface via the cap lamp system and logging out identification. The delay in reporting accidents caused by the current communication system in use can sometimes result in death of the injured victim. Furthermore, according to the Mine Safety Department (MSD), there is currently no software or database based system which has been employed in aiding or speeding the accident reporting or communication system in the mines in Zambia. Therefore, in this study, communications software was developed for underground mines to improve safety communication. This software can effectively and quickly execute the sending of information digitally from underground workings to all the relevant personnel on surface in the shortest possible time so as to enhance the provision of assistance to the affected miners. The software also has an accident reporting and logging database based system which incorporates the digital version of Form 55 used at Ministry of Mines and Minerals Development. In addition, the software provides the last known location of personnel in case of loss of communication with the affected miners and the surface.

At first glance, the developed software may appear to be a replica of the Mine Safety and Health Administration's (MSHA) Personal Emergency Device (PED) system but upon further usage and evaluation, it is in actual sense independent and unique in that it comprises a peculiar functionality that is not housed by the PED and provides a two way communication system. The major difference between the two systems is that, the Mines Safety and Accident Communication System, (MISACOMS) uses a client-server based communication and includes a three-tier architecture model which is comprised of three architectures called the Client tier, the Middle Tier and the Database Tier and provides a conceptual framework for Digital Network database applications. It further employs the data transmission capabilities of Transmission Control Protocol/Internet Protocol (TCP/IP) digital communication which works on Wireless Local Area Networks and also twisted cable networks. The information used in the structuring and understanding of the current Mining Safety procedures, systems and methods of accident reporting was collected from Konkola Copper Mine's (KCM) Nchanga Mines and MSD of the Government of the Republic of Zambia, in Kitwe.

\section{A Review of Underground Communication Systems}

In this section, underground mine communication systems is reviewed. The merits and demerits of each system are further discussed.

Underground mine communication techniques are mainly classified into three categories (Bandyopadhyay et al, 2010) and these are Through the Earth (TTE), through the Wire (TTW) and through the Air (TTA). Apart from these, other techniques are also used for communication and safety purposes which include Carrier current systems and Hybrid systems (Bandyopadhyay et al, 2010). The classification of communication systems is as shown in Figure 1(Rajan \& Sahu, 2014). The short details on communication devices presently used in underground mines for communication and monitoring purposes are given in Table 1. 


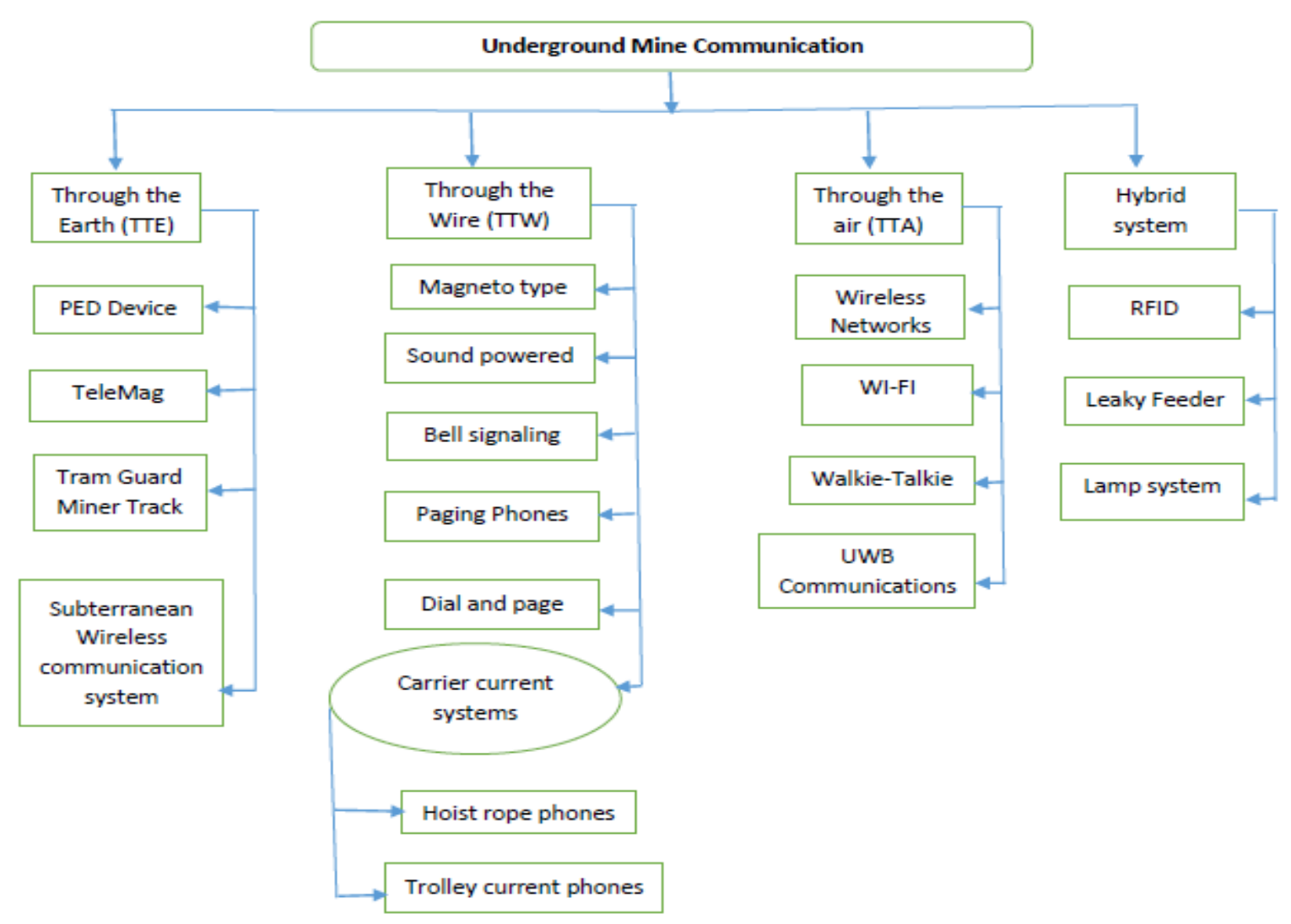

Figure 1: classifications of communication systems ( Rajan $\mathcal{E}$ Sahu, 2014)

\section{Through the Earth (TTE)}

Through the earth communication techniques has been widely researched for the purpose of communication as well as for rescue operation in emergency. Very low frequencies are used to increase the range because the attenuation in Electromagnetic signals decreases with the frequency (Bandyopadhyay et al, 2010). TTE involves a very large transmitting loop antenna managed on the surface of mine. Through the earth communication techniques are used by following communication systems: Personal Emergency Device (PED), TeleMAg, Tram Guard Miner Track and Subterranean wireless electrical communication system. Communication is limited to text messages because the data rate is very poor and operates on these low frequencies (Bandyopadhyay et al, 2010).

\section{Through the Wire (TTW)}

Through the wire (TTW) communication technique provides long distance communication in a routine operation of the mine due to fixed infrastructure (Kennedy et al, 2011). Through the wire communication system works very well in normal mining operation but is highly vulnerable to damage and may lead to entire system breakdown in accidents involving roof falls, fire, and mine collapse and so on. Different kind of electrical conductors used for signal transmission include twisted pair, co-axial cable, and optical fiber etc. Some of communication devices used in mines are: sound powered phones, Magneto type phone, Bell signaling, Paging phones, Dial and page phones, trolley carrier phone and hoist rope phone. Various cable protection schemes involving deployment through borehole connections to main lines, burying the cable and conduit have been implemented to support reliable communication of TTW communication systems (Misra et al, 2010). 
Table 1: Summary of communication devices used in underground mines

\begin{tabular}{|c|c|c|c|c|}
\hline $\begin{array}{c}\text { Communication } \\
\text { System }\end{array}$ & Description & Merits & Demerits & $\begin{array}{l}\text { Communication } \\
\text { Technique Used }\end{array}$ \\
\hline Magneto Phones & $\begin{array}{l}\text { Generates a current that is } \\
\text { strong enough to make bells of } \\
\text { other phones ring in a private } \\
\text { line manner }\end{array}$ & $\begin{array}{l}\text {-Simplicity } \\
\text {-easy to use }\end{array}$ & $\begin{array}{l}\text { - Battery power } \\
\text { requirement } \\
\text {-Modification } \\
\text { requirement with } \\
\text { mine expansion } \\
\text {-Specific coding } \\
\text { requirement to page a } \\
\text { person } \\
\text { - signal strength is poor }\end{array}$ & TTW \\
\hline $\begin{array}{l}\text { Sound-Powered } \\
\text { Phones }\end{array}$ & $\begin{array}{l}\text { Uses the voice of the speakers } \\
\text { and converting them into } \\
\text { electrical signals by electro- } \\
\text { mechanical transducers }\end{array}$ & $\begin{array}{l}\text { - No external energy } \\
\text { source required } \\
\text {-Suitability for rescue } \\
\text { missions } \\
\text {-small in size }\end{array}$ & $\begin{array}{l}\text {-short range } \\
\text {-works as independent } \\
\text { intercom system }\end{array}$ & TTW \\
\hline Paging Phones & $\begin{array}{l}\text { Based on party line fashion. } \\
\text { Each individual } \\
\text { device has its own battery to } \\
\text { feed audio amplifiers which } \\
\text { strengthen the audio signals }\end{array}$ & $\begin{array}{l}\text {-Reliable, } \\
\text { - easy to install } \\
\text {-maintenance simple }\end{array}$ & $\begin{array}{l}\text {-No simultaneous } \\
\text { communication } \\
\text {-Internal battery } \\
\text { required }\end{array}$ & TTW \\
\hline $\begin{array}{l}\text { Dial-and-Page } \\
\text { Phones }\end{array}$ & $\begin{array}{l}\text { Combines the features of } \\
\text { sound powered and paging } \\
\text { systems }\end{array}$ & $\begin{array}{l}\text {-Multiple functionality } \\
\text { (dial and page) in a } \\
\text { single system }\end{array}$ & -installation is complex & TTW \\
\hline $\begin{array}{l}\text { Trolley Carrier } \\
\text { Phone }\end{array}$ & $\begin{array}{l}\text { Based on the connection } \\
\text { between the receiver } \\
\text { and transmitter through the } \\
\text { trolley wire and a } \\
\text { coupler capacitor and operates } \\
\text { at } 60-140 \mathrm{KHZ}\end{array}$ & $\begin{array}{l}\text {-Improved range } \\
\text {-A better insulation } \\
\text { compared to the } \\
\text { telephone systems } \\
\text { - Easy to maintain } \\
\text {-signal with good clarity }\end{array}$ & $\begin{array}{l}\text {-dependent on the } \\
\text { carrier frequency } \\
\text {-Limited coverage } \\
\text {-constant vibration } \\
\text {-quickly warm }\end{array}$ & $\begin{array}{l}\text { Carrier current } \\
\text { system }\end{array}$ \\
\hline $\begin{array}{l}\text { Personal } \\
\text { emergency } \\
\text { device(PED) }\end{array}$ & $\begin{array}{l}\text { Based on ultra-low-frequency } \\
\text { (ULF) transmission that } \\
\text { propagates through rock } \\
\text { strata, }\end{array}$ & $\begin{array}{l}\text { - Reliable } \\
\text {-no dependency on } \\
\text { cables/wires }\end{array}$ & $\begin{array}{l}\text {-one way } \\
\text {-underground to } \\
\text { surface } \\
\text { communication is not } \\
\text { possible }\end{array}$ & TTE \\
\hline $\begin{array}{l}\text { Ultra-Wide Band } \\
\text { Systems }\end{array}$ & $\begin{array}{l}\text { Based on narrow pulses and } \\
\text { requires very low energy }\end{array}$ & $\begin{array}{l}\text {-High data rate with low } \\
\text { power } \\
\text {-Very high accuracy in } \\
\text { location tracking } \\
\text { applications } \\
\end{array}$ & $\begin{array}{l}\text {-Short range due to low } \\
\text { power }\end{array}$ & TTA \\
\hline Walkie -Talkie & $\begin{array}{l}\text { Wireless communication; uses } \\
\text { ultra-high frequency } \\
\text { (UHF)with an antenna at the } \\
\text { top of the system }\end{array}$ & $\begin{array}{l}\text { - handheld } \\
\text {-portable with two way } \\
\text { communication }\end{array}$ & $\begin{array}{l}\text { Generally poor range } \\
\text { but may have good } \\
\text { LOS performance }\end{array}$ & TTA \\
\hline WI-FI & $\begin{array}{l}\text { It uses radio frequency to } \\
\text { transmit data through air and } \\
\text { based on IEEE } 802.11 \\
\text { standards. }\end{array}$ & $\begin{array}{l}\text {-two way } \\
\text { communication } \\
\text {-supports voice and data } \\
\text { communication }\end{array}$ & $\begin{array}{l}\text {-limited coverage } \\
\text {-poor traffic } \\
\text { management } \\
\text {-power consumption } \\
\text { is high }\end{array}$ & TTA \\
\hline $\begin{array}{l}\text { Leaky feeder } \\
\text { system }\end{array}$ & $\begin{array}{l}\text { Signals leak over the entire } \\
\text { length hence, prevents from } \\
\text { external signal interference }\end{array}$ & $\begin{array}{l}\text {-mobile communication } \\
\text {-reliable } \\
\text {-two way voice and data } \\
\text { communication } \\
\text {-capable for video } \\
\text { communication } \\
\end{array}$ & $\begin{array}{l}\text {-limited range } \\
\text {-weak performance } \\
\text { where NLOS }\end{array}$ & Hybrid \\
\hline
\end{tabular}




\section{Through the Air (TTA)}

Through the Air (TTA) communication technology has drawn a significant attention of researchers and different manufacturers across the globe. This is so because of the increasing need of infrastructureless communication systems and reliability in emergency scenarios. These techniques use a wireless link for signal transmission. Underground mine environment either in coal mines or metal mines creates a very big challenge for wireless communication. This is because an underground mine has different characteristics for signal propagation that affect the performance of communication systems. Wireless networks, Wi-Fi, Walkie-Talkie, Utra wideband (UWB) communications are classified into the categories of through the air (Bandyopadhyay et al, 2010). The limitations of TTW communication forced the mining industry to have TTA communication technology which is more reliable, easy to maintain and economic in comparison to TTW communication systems.

\section{Hybrid Systems}

The hybrid system possesses the advantages of both through the wire (TTW) and through the air (TTA) communication systems. This leads to the better coverage range of communication systems for underground mines. Hybrid systems approach can further extend to forward or send and receive signals to a node using wireless mesh network upon short wireless link. Leaky feeder system and Radio Frequency Identification (RFID) based systems are now widely used in mines to achieve two way communications. This kind of deployment of hybrid system has the option of multipath for transmitting and receiving which are in the coverage range to each other (Misra et al, 2010). Hybrid systems support both voice and video communication in underground mines with high data rates (Misra et al, 2010).

\section{Recent developments in communications and safety system for underground mines}

Research in development of a reliable and robust communications and safety system for underground mines has continued. Misra et al (2010) experimented for assessment of commercially available wireless sensor nodes for wireless communication in underground mines. The tests were carried out in gold and copper mines in Australia. The results suggested that the success rate is high of those nodes which had clear line of sight (LOS) and placed near to the base station. A "Zonal Location Tracking "system has further been developed for tracking mine personnel, vehicles and plant in underground mines. The developers used ZigBee Pro Mesh wireless networking standard based system comprises of ZigBee router (ZR) devices installed at known locations. The system had 40 mobile nodes to support a large enough network. Results had shown that to capture all the mobile devices, polling interval should be ten seconds or less for a nominal distance of 50m (Kennedy et al, 2011). Going forward, Bandyopadhyay et al. (2011) developed a wireless safety system for underground mines. The system had ZigBee complaint active Radio Frequency Identification (RFID) devices as a core component which was based on wireless mesh network infrastructure. The system was tested that packet delivery ratio is inversely proportional to the distance between end nodes to coordinator. Furthermore they have also concluded that number of hops also affected the packet delivery ratio. Kumar et al (2011) developed a communications system using wireless nodes for underground mines. The system was developed using MSP430 microcontroller and nRF24L01 radio transceiver operating on $2.4 \mathrm{GHz}$ license free band and consumed very low power. Although authors initially reported good results in lab but real time implementation are yet to be performed. Zhu et.al (2009) designed and analysed the system performance of the developed system considering various communication parameters and safe operation requirements. The results obtained from the tests of his design in virtual mine revealed that the maximum communication delay of the network is about 0.11 seconds and the maximum packet loss rate is about 0.13 . These results assured the reliability of the system. However, neglecting other critical characteristics of the underground wireless channel is still an issue to consider for performance evaluation of the proposed system. Chehriet.al (2010) in his research carried an experiment at MMSL-CANMET laboratory mine in Canada. They used IEEE802.15.4 ZigBee based platform for real time implementations. They concluded their work stating 
that behavior of radio transmission in underground mine tunnel is far away from an ideal normal environment for wireless communication. And also the geological structures of mine galleries acted as scatters which caused for fading in signal strength at receiver (Chehri et.al, 2010).

Ranjan et.al (2015) proposed an enhanced hybrid channel model with loss due to surface roughness for mine tunnel roadways based on the concept of multimode wave propagation. He further studied and described a new hybrid multimode model for wireless communication applications in underground coal mines; analyze it with respect to significant parameters such as operating frequency, mine tunnel size, transmitter-receiver position, electrical parameters and antenna gain. The characterization results suggest that the mine tunnel size and transmitter-receiver position affects the signal behavior significantly (Rajan et al, 2017).

\section{A brief review on database management systems}

A data base management system (DBMS) is a collection of programs that enables you to store, modify, and extract information from a database. There are many different types of database management systems, ranging from small systems that run on personal computers to huge systems that run on mainframes (David \& Huge, 2004). A database might contain a few entries that make up a simple address book of names, addresses, and phone numbers. At the other extreme, a database can contain tens or hundreds of millions of records. Most database applications have small to medium size databases that store thousands, or tens of thousands, of records.

The database server applications interface is accessed using Structured Query Language (SQL). It's a standard query language that's used to define and manipulate databases and data, and it's supported by all popular database servers. SQL also allows you to update and delete data and databases, and it includes many other features such as security and access management, multiuser transactions that allow many users to access the same database without corrupting the data, tools to import and export data, and powerful undo and redo features (David \& Huge, 2004).

\section{METHODOLOGY}

\section{The software Algorithm}

The software's algorithm is as indicated in Figure 2. The client application will be programmatically configure to client gadgets that shall be connected wirelessly or via a hardened Local Area Network (LAN) cable network. The gadgets will each contain a peculiar and unique serial number that will be used in the identification of the team, section, leadership and location of the miners to whom it will be assigned.

The client software is to be developed in such a manner as to constantly remain connected to the server software, provided the Internet Protocol (IP) address remains static, and set to issue an alarm/warning in the event that this connection is lost. This alarm is simultaneously issued on the server indicating that a client, with serial "abc", has lost connection with the server at such a moment.

MISACOMS' database can then be referenced against the given serial in order to identify and know the exact details of the gadget from which the signal was lost. This information can be used to prepare in an emergency situation and also in confirming the severity of the situation by trying to re-establish connection or sending a message to the client and waiting for a response. 


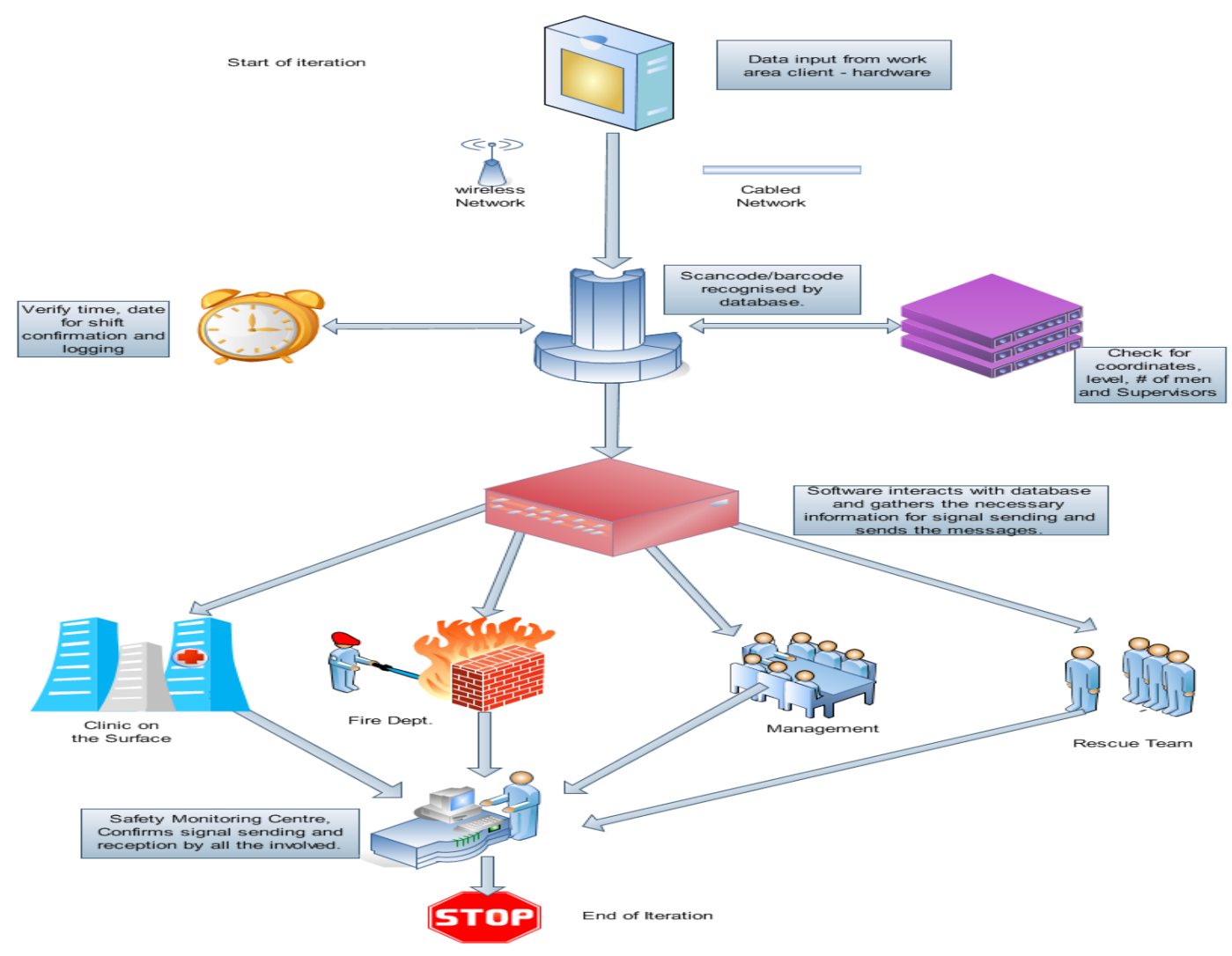

Figure 2: MISACOMS Algorithm

Resident on the client application is a means to both read and send texts to the server application that will equally be able to receive and reply accordingly. It is this platform from which the gadget with the client application will use to send the full details if and when there is need for emergency rescue services underground. The client is shown in Figure 3

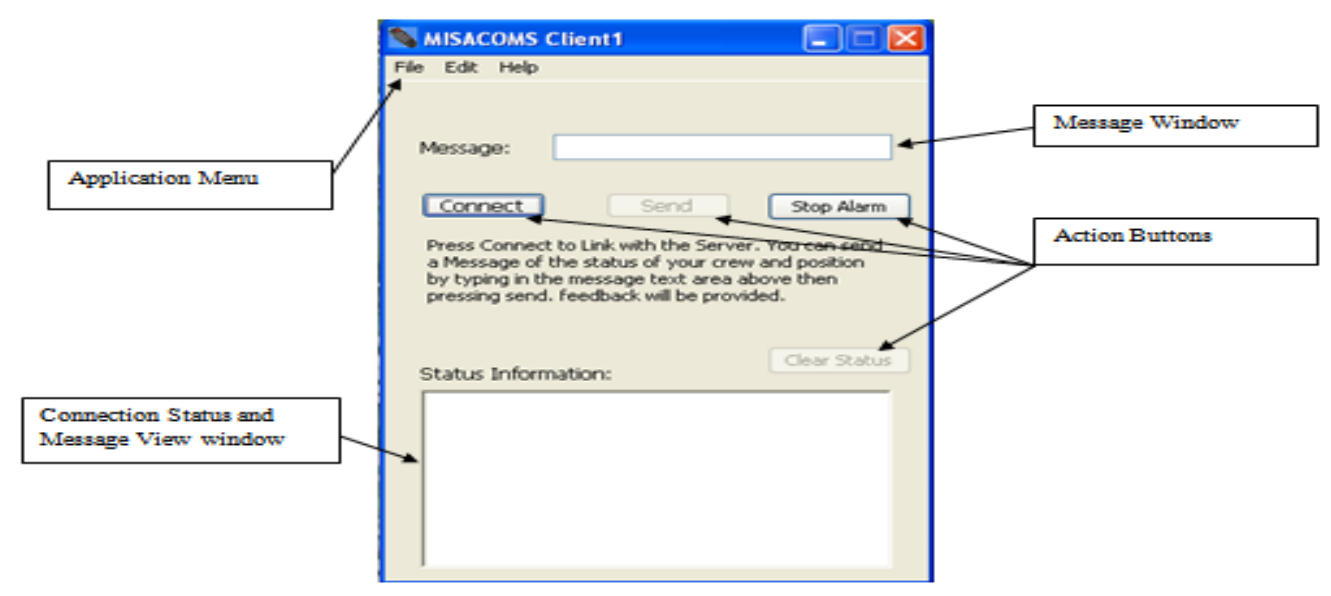

Figure 3: Graphical User Interface (GUI) on the Client Software as seen on Windows XP

The server's GUI is similar to that of the client with the difference in the functionality of the action buttons as shown in Figure 4. 


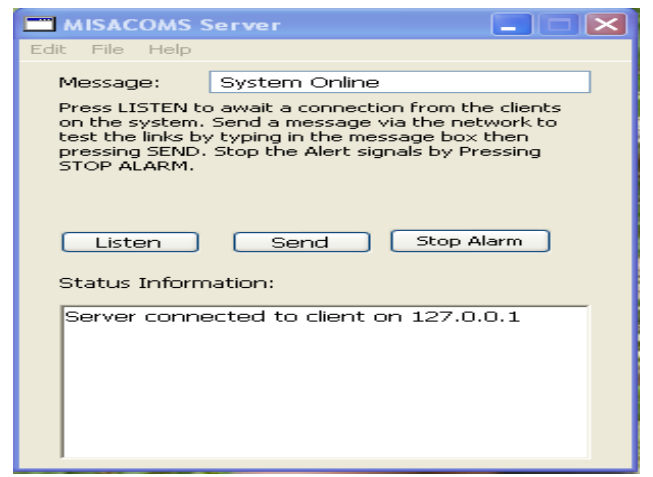

Figure 4: Server Application's Graphical User Interface

The server is to be installed on a computer alongside the Logging and Reporting component of MISACOMS. This should be on the surface or stable environment where no disturbances can or will frequently occur. The people monitoring this robust system must be available at all time to respond to the queries that might be coming in from underground, cross-reference the database and respond accordingly.

The server application works in a similar manner to the Client application though it only listens for connections that will be incoming from the various gadgets running the client application on the site. It also has the capacity to detect a disconnection from a particular client whose information it will show and provide cross-referencing details for the person(s) monitoring. The Server application must be installed on a computer with a static and not a dynamic IP address so as not to confuse that which will be input in the gadgets as this will cause them to loss connection which will only be restored should the IP addresses in the tally.

\section{Development Mines Safety and Accident Communication Software}

The software was developed using a programming language called Real Basic, an object-oriented, invent driven Language.

\section{Activities for Designing the Software}

1. Design of the user graphical interface for both the client and server software

- The client application is the one that shall be on the production front or on the person of the mine working in that particular area. This will be assigned a unique identification which shall differentiate it from the other clients that will be deployed in the mine. This unique id will be checked against data stored in the database to provide information on whom and where it is being used.

- The interface shall have a means by which a miner can request for a remote connection to the server and also read what responses will be sent from there. Upon reception of the message, an alarm will go off to signal receipt of a notification from the client.

2. Setting up of unique IP/TCP communications ports for software to software communication

3. Design and develop the client software which shall be installed on the computers to act as the gadgets that will be placed in the production front.

4. Design and develop the server software which shall be placed in the main control room, to constantly monitor which client triggers an alert or gets disconnected abruptly. 


\section{Accident and Data Logging System}

The software will be developed using programming languages called RealBasic (RB), Hypertext Preprocessor (PHP), Extensible Hypertext Markup Language (HTML), Cascading Style Sheets (CSS), JavaScript, Action Script and Structured Query Language (MySQL), a database management, design and maintenance language.

\section{Activities for Designing the Software}

- Design the table layout for the database to be used in the application

- This will involve a design of the data fields in which the data shall be stored and also setting the data types and unique ids.

- Design and develop the application's graphical user interface, form fields.

- Create the interface from which the users tasked to monitor, report and log incidents shall interact with the software. It will be design to be an easy to use interface with basic buttons, data entry fields, e.t.c.

- Incorporate mine safety department (MSD) 55 parameters, by creating a tabbed interface from which the data will be written directly into the database.

$\circ$ The digital version of form 55 will be incorporated into the software and its interface and graphical platform will enable easy data entry into the database.

- Design a search feature that will enable the application to query the database and provide dynamic information as per requirements.

\section{RESULTS AND ANALYSIS}

The following illustrations show the results that were obtained during the testing and trials of the responses between the server and the client.

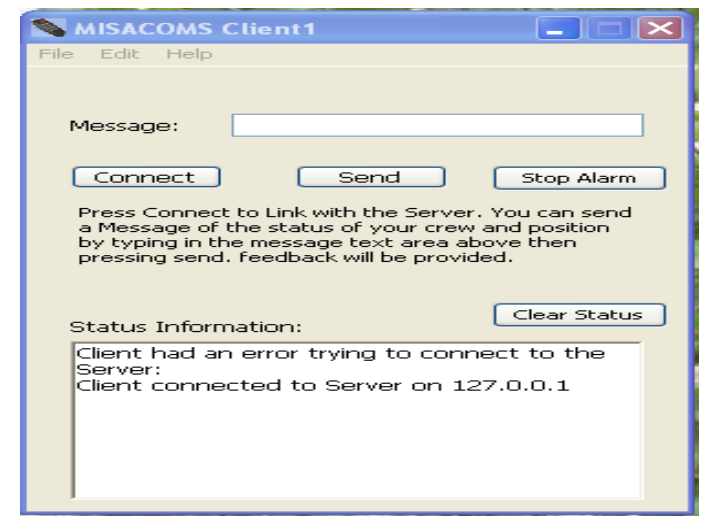

Figure 5: Client application attempting connections to the server application.

The information in the status box of Figure 5 shows two results. The first being the failure of the client application to identify and connect to the server application and the second showing a successful connection. The causes in the failure of the connection were:

a. Incorrect Internet Protocol Address: assigning a different or incorrect IP Address to the client made it impossible for the client application to locate and connect to the server.

b. Server not switched on: the server application was not initiated or switched on.

c. Server not Listening for incoming connection requests: the server application was switched on but was not in the listening state. That is the state in which it is supposed to be listening for incoming connection requests that are sent from the client application.

d. Server has a dynamic IP address; in the event that the server application is installed on a machine with a dynamic IP Address, the address changes every time the computer is switch on 
as these are automatically assigned based on the availability of the addresses. This unexpected change in the IP address will cause the client application to look for the server connection on the particular IP address which it was configured to try and connect to.

In the event that all the information is correct and there is no broken LAN/WLAN connection between the gadget and the server, the connection is successful and all the other communications between the two can commence.

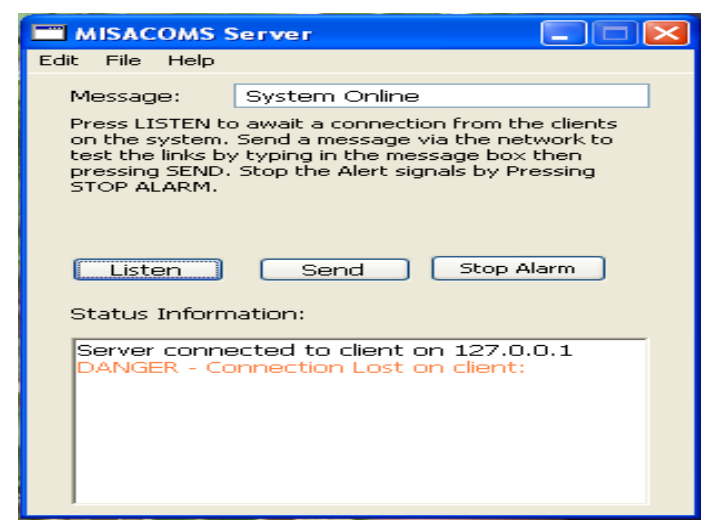

Figure 6: Server Application, online and Connection lost

The results shown in the status box of Figure 6 show a successful connection to the client application and the other (in red) shows a lost in connection to the client.

The loss of connection to a client application can be caused by any of the following;

a) Gadget not switched on: the server application was not initiated or switched on.

b) Server's Listening function was reset: the server application was switched on but while it was connected, the listen button was pressed. This causes the server to abort all connections and start listening for new ones.

c) Loss of communication between the client and server: If all the parameters are correctly set, this is usually an indication that something has gone wrong and appropriate measure need to be taken.

In the event of a reported sudden loss of communication, it could mean that the connection was severed by a cut in the LAN/WLAN infrastructure connecting the client and server application. This could be as a result of rock fall, cable snapping or an explosion that would warranty an emergency response in the event that communication is not re-established.
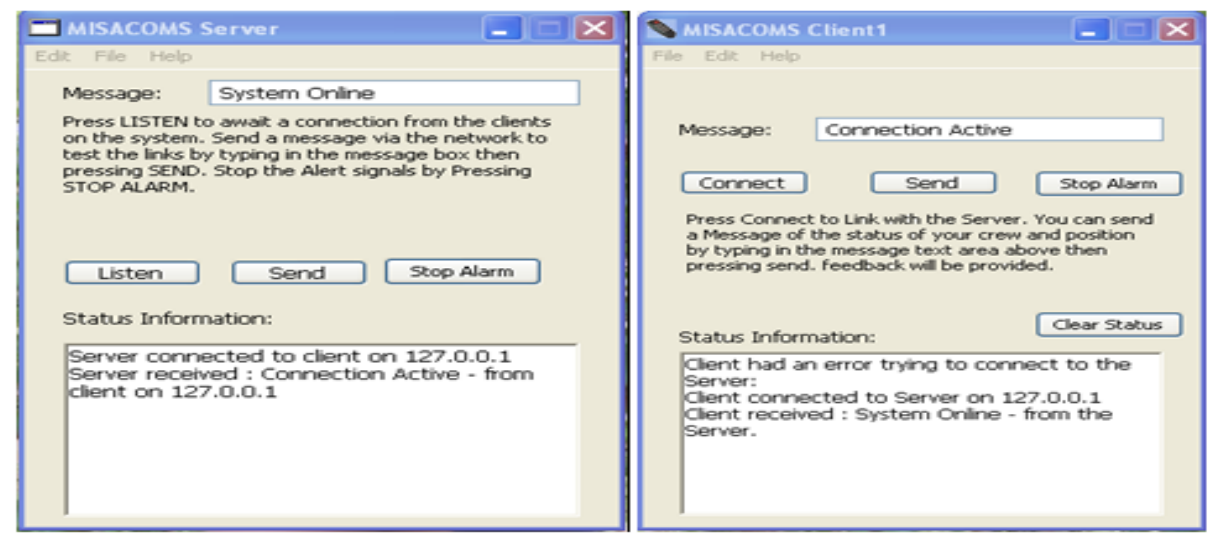

Figure 7: Server-Client Messaging Capabilities 
Figure 7 shows a successful transmission of text based messages between the client software and server application. This can and will be used to relay vital information should there be impending danger on the site or the occurrence of an accident underground.

The MISACOMS Logging and Reporting system, incorporating the digital version of Form 55 is a webclient (browser) based application which had the capability to enter, search, update and delete records from the database. Being developed in a top, Web3 languages, the application was able to function across different operating system platforms as long as the dependency files and local or online server systems are available. It was able to run on Windows based platforms and Linux systems like Ubuntu, Mint and Red Hat. It was not tested on any Mac based operating systems but the functionality would still be as was observed on the other operating systems.

\section{DISCUSSION}

The software was tested on a single thread network in a computer lab at school of mines of mines university of Zambia. However, for a more robust experience, the project should be tested on multithreaded server application which can take on an unlimited number of client connections. This could have provided a scenario from which to trouble shot in the event of unseen hindrances resulting from multi-thread requirement. The computer on which the server application will be installed must have a static IP address. This is to avoid the issues of a conflicting IP address, loss of connection with the client application and the inability of the client to locate the server. The client software is designed to check for a particular IP address on the LAN/WLAN and a particular communications port which the server software will designate to be listening for incoming communications from the clients. Network setup of either wireless or cable can be used. The choice of the network will largely depend on the conditions prevailing underground. Wireless connections will require additional aerials and router to be installed underground so as to boost the transmission of the signal from underground to the servers on the surface. Cable networks on the other hand can run straight from the surface to the point of operations underground and can be easily moved to a new location if development and exploitation of the mineral resource is completed. The hardware to makeup the gadget and it requirements will equally depend on the preferences of the mines. The hardware can be designed for individual or crew use. The MISACOMS Reporting and Logging was developed and based on 3-tier architecture was broken down into database tier, middle tier and client tier. The application requires an offline server to be installed and configured accordingly in order for it to properly function and link all the scripts.

\section{CONCLUSIONS}

The communications software was developed, and tested on a virtual and actual server client networks. It can provide a two way texting and chatting system which is useful in the relaying of information between the surface and teams working underground. The clients to be deployed underground will have a unique id which can be referenced to give details on the locations. This can further ease the search for the miners in case of total loss of communication and accidents

The MISACOMS Reporting and Logging system was developed and tested on various operating systems with the following being observed: (1) The digital format of Form 55 provides a easy way of logging, reporting and cross referencing data as it is stored in a database. (2) The web-like platform of the application gives ease of navigation to all the other details that may be needed to be reference with the server application of the accident monitoring systems. (3) The Observed time lapse between the time of sending the message and/loss of connection between two different gadgets was $3.5 \mathrm{~ms}$ to $6 \mathrm{~ms}$ depending on whether the gadget was using a wired connection $(3.5 \mathrm{~ms})$ or a wireless connection $(6 \mathrm{~ms})$.

The software will have to be tried at various underground mine in Zambia. The hardware to makeup the gadget and its requirements will depend on the preferences on the mines. It can be designed in such a 
manner as to be used by one person or a group of individuals working in a team. It can also be designed to be incorporated in the machinery used for all mining operation.

With the evolution of technology and surfacing of microcomputing interfaces like those use in the Internet of Things, the MISACOMS system has great potential in being a cheaper, safer and efficient communication system. Incorporated geolocation tied to inbuilt GPS on either an Arduino, Galileo or Raspberry-Pi micro-controller would ease the location tracking by providing exact data on a 3dimensional plane. This would further make it easier and simpler to create a simple network underground without requiring additional customized infrastructure that would survive the harsh conditions of working undergound.

\section{REFERENCES}

Bandyopadhyay, L.K., Chaulya, S.K. and Mishra, P.K. (2010) Wireless Communication in Underground Mines, RFID Based Sensor Networking, Springer Publications, New York

Bandyopadhyay, L.K., Chaulya,S.K. Mishra, P.K., Dutta, S., \& Shukla,S. (2011) Wireless Safety System for Underground Mines, 34th International Conference of Safety in Mines Research Institutes, pp.655-668, Macmillan Publishers,

Chehri, A., Mouftah H., Fortier, P., \& Aniss H. (2010) Experimental Testing of IEEE801.15.4/ZigBee ${ }^{\mathrm{TM}}$ Sensor Networks in Confined Area. 8th Annual Communication Networks and Services Research Conference, IEEE Communication Society, 244-247

David, L., \& Hugh E. W. (2004) Web Database Application with PHP and MySQL, 2nd Edition, O'Reilly Media, Inc., ISBN: 0-596-00543-1.

Kennedy, G., Bedford, M., Foster, P., \& Jones, B. (2011) Underground Tracking and Positioning Techniques Using Wireless Mesh Networking Technology, 34th International Conference of Safety in Mines Research Institutes, pp.611-624, Macmillan Publishers.

Kumar,V., Sonavane,S.S., \& Patil,B.P. (2011) Wireless Sensor Network for Coal Mines, 34th International Conference of Safety in Mines Research Institutes, pp.655-668, Macmillan Publishers,

Misra, P., Kanhere, S., Ostry, Diethelm, Jha, \& Sanjay (2010) Safety Assurance and Rescue Communication Systems in High-Stress Environments: A Mining Case Study, Topics in Design\& Implementation, IEEE Communications Magazine, pp. 66-73.

Ranjan, A., \& Sahu, H.B. (2014) Adancements in communication and safety systems in underground mines. Present status and future prospects, proceedings of Zaytoonah University International conference on design and innovatiom in sustainability.

Ranjan, A., Sahu, HB., \& Misra P. (2015) Wave propagation model for wireless communication in underground mines, - Bombay Section Symposium (IBSS), IEEE,

Ranjan, A.,Misra,P., Dwivedi, D., \& Sahu HB. (2017) Studies on Propagation Characteristics of Radio Waves for Wireless Networks in Underground Coal Mines, Wireless Personal Communications, Underground Communications \& Tracking Systems Update,

Zhu, C.G., Chun-F.S., \& Li-J.Z. (2009) Design and performance analysis of wireless sensor network location node system for underground mine. Elsevier, Mining Science and Technology19, 0813-0818. 
